\section{Xeroderma pigmentosum with ocular involvement and squa- mous cell carcinoma: A case report}

\author{
Bernadya Yogatri Anjuwita Saputri, \\ Iskandar Zulkarnain \\ Department of Dermatology and \\ Venerology, School of Medicine, \\ Universitas Airlangga, Dr. Soetomo \\ General Hospital, Surabaya, Indonesia
}

\begin{abstract}
Xeroderma Pigmentosum is a rare, autosomal recessive genetic disorder, characterized by defective DNA repair leading to clinical and cellular hypersensitivity to ultraviolet radiation and carcinogenic agents. Patients with xeroderma pigmentosum often have cutaneous and ocular sun sensitivity and freckle-like skin pigmentation. Skin changes are the most important symptoms and they manifest as erythema, painful blisters and ulceration. The last level of skin changes transformation are malignant neoplasms, particularly squamous cell carcinoma. There is a great involvement of many parts of the body, especially the head and neck. This paper describes a case of xeroderma pigmentosum with advanced cutaneous squamous cell carcinoma and ocular lesions in a nine-year-old boy. The extensive ultraviolet radiation-induced skin and eye damage are evidence of a failure to use sun-protection and a lack of appropriate medical care from childhood.
\end{abstract}

\section{Introduction}

Xeroderma pigmentosum (XP) is a rare, inherited autosomal recessive condition. It is characterized by photo-hypersensitivity of tissues exposed to sun and by a several thousand-fold increase in the risk of developing malignant neoplasms of the skin. Mutations in xeroderma pigmentosum DNA genes that regulate nucleotide excision repair not only predispose persons with xeroderma pigmentosum to malignancies and tumors such as squamous cell carcinoma, but also promote premature cutaneous and ocular ageing. Clinically apparent ocular diseases include eyelid atrophy and tumors, corneal sicca and opacification, exposure keratitis, pterygium and chronic conjunctival injection. We report a case of xeroderma pigmentosum in a young boy, co-existing with squamous cell carcinoma of the neck.

\section{Case Report}

A nine-year-old boy was presented to our outpatient clinic with a history of skin pigmentation over his face, neck and trunk, and photophobia since nine months of age. The pigmentation initially appeared on his face then spread out to include his forehead, neck and upper trunk, as well as both arms and legs. The pigmentation was progressive and worsened after exposure to sunlight, particularly in overexposed areas. The patient had experienced redness and watering in his eyes for about a year prior to hospitalization, and in the final two weeks before hospitalization it was getting worse (Figure 1A). He was also finding it difficult to close his eyes properly and complained of photophobia.

There was ulceration on the posterior of the neck side, present for eight months (Figure 1E). At first, the ulceration looked like a papule, then it became larger. Local examination revealed extensive ulceroproliferative lesions on the posterior of the neck side with a $7 \times 7 \mathrm{~cm}$ area of surface redness. There was some slough with haemorrhage, sharply marginated, which bled on contact.

There was consanguinity in his family, his mother mother marriage with his cousin (his father), this pedigree of this family presented in Figure 1F. On general examination, the patient was pale and underweight for his age. A detailed history revealed similar symptoms in his sibling aged one year. The child was immunized with no significant perinatal history. The development of milestones was normal. This patient suffered from poor nutrition and his body weight was less than normal for his age.

Multiple pigmented lesions and skin freckles were noticed all over his body (Figure 1B-1D). There was no cervical lymphadenopathy. Systemic examination including neurological functions was essentially normal. Moreover, ophthalmological examination revealed photophobia with decreased visual acuity, and corneal scarring. Examinations of the anterior segment and fundus were difficult to evaluate. A cytology examination showed features that moderate dysplasia squamous cell (Figure 2B). Haemogram and serum biochemistry were within normal limits. A pathological examination of a fragment of the tumor on the patient's neck was confirmed to be squamous cell carcinoma (Figure 2A). Based on skin histopathology, a diagnosis was made: xeroderma pigmentosum with atrophy, hyperplasia of melanocytes with melanin in epidermis. In a
Correspondence: Bernadya Yogatri Anjuwita Saputri, Department of Dermatology and Venerology, School of Medicine, Universitas Airlangga, Dr. Soetomo General Hospital, Surabaya, Indonesia,Prof Moestopo 47 street, 082180146143

E-mail: gatrianwar22@gmail.com

Key words: xeroderma pigmentosum, squamous cell carcinoma, pigmentary retinal degeneration, genetic skin disorder.

Contributions: the authors contributed equally.

Conflict of interest: The authors declare no potential conflict of interest.

Received for publication: 1 February 2019. Accepted for publication: 22 February 2019.

This work is licensed under a Creative Commons Attribution-NonCommercial 4.0 International License (CC BY-NC 4.0).

(C) Copyright B.Y.A. Saputri and I. Zulkarnain, 2019 Licensee PAGEPress, Italy

Dermatology Reports 2019; 11(s1):8082

doi:10.4081/dr.2019.8082

multidisciplinary meeting (pediatric oncology, ophthalmology and dermatology), the decision was made to perform neoadjuvant chemotherapy treatment for tumor mass reduction. This patient gets six cycles of chemotherapy and has to go through a period of two cycles.

\section{Discussion}

Xeroderma pigmentosum (XP) is a rare genetic photosensitive disorder in patients showing susceptibility to skin cancers where the body is exposed to the sun. It was first described at the end of the 19th century by Moritz Kaposi, a dermatologist who described a severe photosensitivity disorder that was accompanied by pigment change. ${ }^{1}$

Ultraviolet (UV) irradiation is composed of UV A spectrum and UV B spectrum; UVB plays an important role in the etiology of XP. UV irradiation causes photoproducts in DNA, chiefly cyclobutane pyrimidine dimers (CPDs) and 6-pyrimidine-4-pyrimidone, which further brings about cell death, mutagenesis, carcinogenesis and cellular ageing. ${ }^{2}$

$\mathrm{XP}$ is an autosomal recessive disorder resulting from mutations in any of the eight genes. These genes restore the DNA damage induced by UV radiation by a process known as nucleotide excision repair (NER). XP patients have mutations in one or more NER genes, which cause molecular defects in cellular DNA repair mechanisms and hypersensitivity to UV radiation. As a 
result, the accumulation of unrepaired UVinduced DNA damage occurs, which either facilitates cell death - contributing to accelerated skin ageing - or cellular transformation resulting in the development of malignancies. $^{3}$

Children with Xeroderma Pigmentosum develop multiple cutaneous neoplasms at a young age. Two important causes of mortality are metastatic malignant melanoma and squamous cell carcinoma, this condition being caused by ultravioletinduced skin hypersensitivity. Another result of ultraviolet exposure is the possibility of degenerative and proliferative cutaneous changes, including hyperpigmentation, skin atrophy, poikiloderma and actinic keratosis. ${ }^{4}$

Clinical manifestation xeroderma pigmentosum passes through three stages. The skin is healthy at birth. Typically, the first stage makes its appearance after the age of six months. Diffuse erythema, scaling, and freckle-like areas of increased pigmentation characterize this stage. The second stage is characterized by poikiloderma, giving rise to an appearance similar to that of chronic radiodermatitis. The third stage is heralded by the appearance of numerous malignancies, including basal cell carcinoma, squamous cell carcinomas, fibrosarcoma and malignant melanoma. ${ }^{5}$ This patient had already trough all the stage, with malignancy appearance.

Skin features of xeroderma pigmentosum include the marked freckling of sun-exposed areas; this can appear before the child is two years old. Another symptom is photosensitivity approximately $50 \%$ of XP patients show acute sun sensitivity such as sunburn occuring after minimal sun exposure; this is often noticed during infancy. Xerosis skin (dry skin) is also common. From neurological features, there are $30 \%$ xeroderma pigmentosum patients that have neurological manifestations such as acquired microcephaly, diminished or absent deep tendon stretch reflexes, progressive sensori neural hearing loss, and progressive cognitive impairrment and there are no neurological complications for these patients. ${ }^{3}$

Histopathologically, patients with xeroderma pigmentosum show hyperkeratosis and increased melanin pigment (this corresponds to the clinical freckling) in the basal cell layer. Some rete ridges may be elongated, whereas other rete ridges may be atrophic. These findings may be accompanied by a chronic inflammatory infiltrate in the upper dermis. In the second stage, atrophy, hyperkeratosis, hyperpigmentation and telangiectasia may be prominent. In addition, the epidermis may exhibit architectural disorder and atypia, and the dermis may be elastotic. The histologic appearances of the various tumors that complicate Xeroderma Pigmentosum are seen in the thrid stage of XP. ${ }^{6}$

The most common types of cancer found in XP patients are basal cell carcinoma and SCC, mostly involving the face, head and neck. SCC in the scalp tends to have an aggressive course. Because of the anatomical structure and the vascularity of the scalp, SCC in this part of the body can extend beyond what is clinically apparent. Cutaneous neoplasm in XP patients is difficult to prevent. However, early protection from ultra-violet (UV) radiation may play a role in the prevention of skin malignancy. Individuals with XP should strictly avoid sun exposure; they should use appropriate clothing, sunblocking agents and protective sunglasses. In addition, early diagnosis of any skin lesion is of the utmost importance. Followups on a regular basis are critical to detect and excise pre-cancerous lesions and malignant tumors at an early stage.?

It is common for persons with XP - and this includes our patient - to have ocular abnormalities caused by ultraviolet-induced DNA alteration to epithelial cell conjunctiva, the cornea, and the eyelid. Also common are photophobia, conjunctivitis, and keratitis that may lead to leukocorneal, hyperpigmentation of the eyelids, loss of eyelashes and malignancies, including
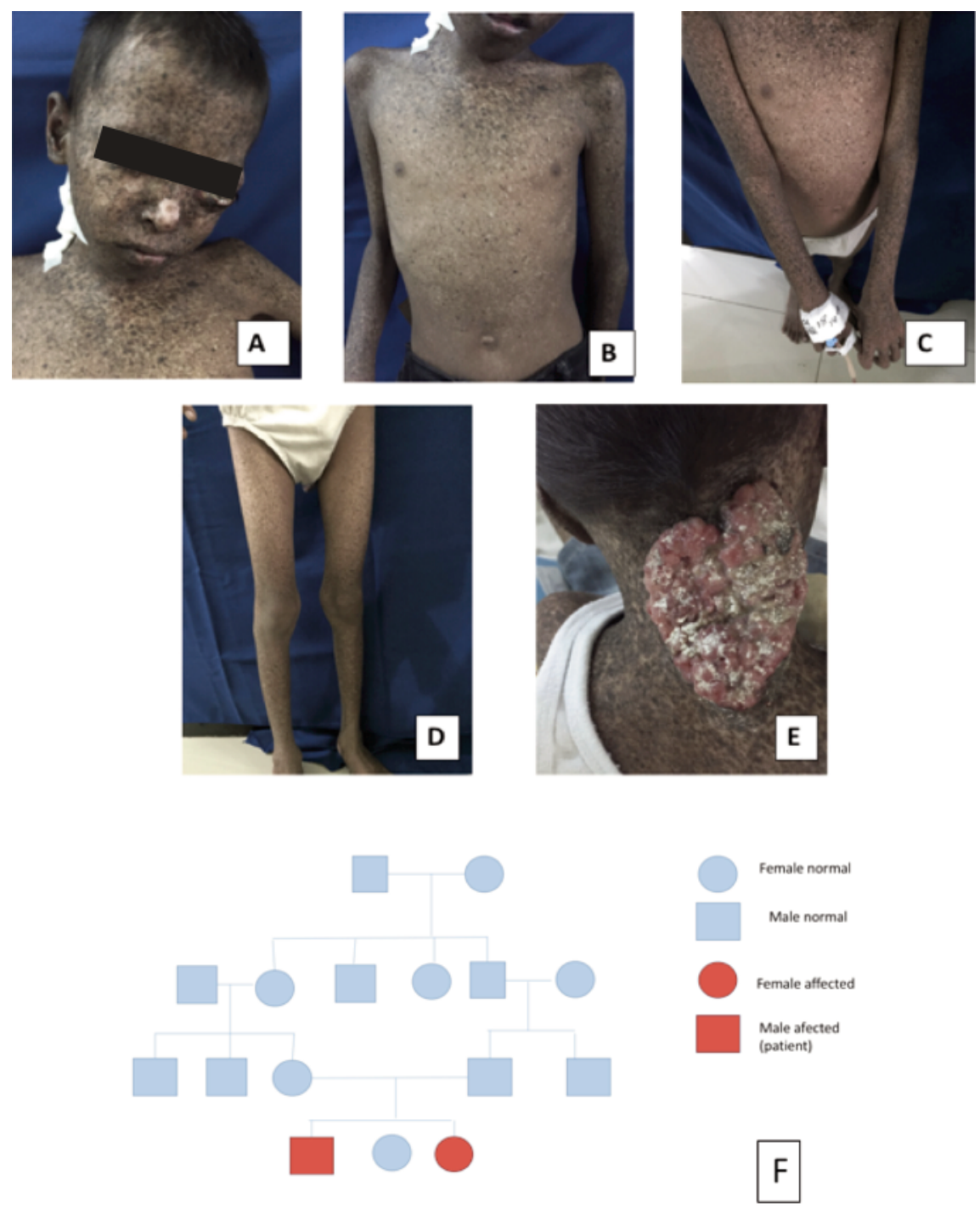

Figure 1. A. face showing numerous freckles and proliferation in left eye. B-D. Hyperpigmented and hypopigmented macules papules in the trunk, extremitas superior and inferior. E. Large ulcer squamous cell carcinoma over his neck. F. Picture of pedigree from this patient show that his parent did consanguineous marriage. 

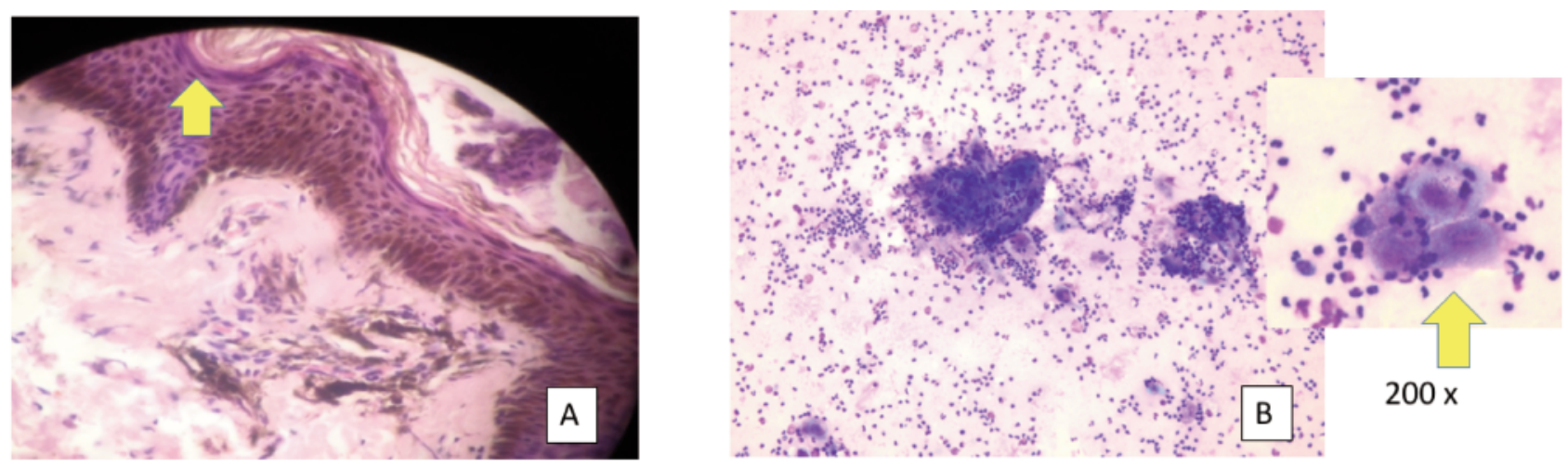

Figure 2. A. Skin Biopsy Athropy,hyperplasia of melanocytes with melanin in epidermis,dilated blood vessels with lymphocytes infiltration and some macrophages that contain melanin in dermis. This result concordance with Xeroderma Pigmentosum. B. FNAB result showing anaplatic squamous cell with atypical cell and large nucleus.

SCC, basal cell carcinoma and melanoma. ${ }^{8}$

This patient also affected in superior eyelid with moderate squamous cell carcinoma. There are three ways in which the eyes can be affected in XP: (1) UVR exposure resulting in DNA damage to the eyelids and periocular skin; (2) UVR exposure resulting in DNA damage to the ocular surface; and (3) the ocular manifestations of neurodegeneration. Even patients with few ophthalmic signs commonly describe photophobia, which is the earliest presenting ophthalmic symptom of XP. Damage to the eyelids and periocular skin can result in the development of cicatricial skin changes as well as skin cancers, which require excision. ${ }^{9}$

No consistent routine lab abnormalities are present in XP. The diagnosis is based mostly on clinical findings and biopsy analyses. However, many studies can be performed in specialized laboratories to help diagnose the condition. These studies include cellular hypersensitivity to UV radiation, chromosomal breakage studies and complementation gene sequencing to identify the specific gene complementation group. ${ }^{10}$

Curently, there is no specific treatment for xeroderma pigmentosum. The treatment of XP is challenging because it is a multiorgan and multisystem disease and, usually, a significant amount of tissue damage has already occurred by the time of diagnosis. Malignant tumours may have already developed in the third or fourth year of life. An early diagnosis and immediate implementation of rigorous sun protection measures may prolong the lives of persons with XP. About two thirds of unmanaged subjects die before the age of 20 years but, as reported by Cleaver and Revet, in climates with intense sunlight exposures, children with XP who do not implement sun-protection measures and have limited access to modern medical care have a shorter life expectancy of about 10 years. ${ }^{5}$

Persons with xeroderma pigmentosum must avoid exposure to any source of sunlight, fluorescent, halogen and mercuryvapour lights, and they must wear protective clothing and UV-absorbing eye glasses. They must also use high-protection factor sunscreens. XP associated cutaneous, ocular and oral lesion disorders should be treated as in any other person. A topical application of 5-fluorouracil or imiquimod is appropriate for premalignant and surgical excision for malignant neoplasms of the skin, tongue, eyelids, conjunctiva and cornea. Methyl cellulose or quinodinecontaining eye drops, and bland ointment at night, constitute correct eye-care. ${ }^{11}$ We treat this patient with sunblock and emolinet, and for squamouscell carcinoma we did chemotherapy not surgery because this patient suffer poor nutrition and the condition not good enough for surgery.

It must be remembered that persons with XP who are properly protected from sunlight may suffer from vitamin D deficiency as a consequence; they should therefore routinely take vitamin D supplements. ${ }^{5}$

DNA repair leads to clinical and cellular hypersensitivity to ultraviolet radiation. General features of the disease include parental consanguinity, onset of symptoms in the first two years of life, pigmentation or freckles in the sun-exposed parts of the body and later, the development of premalignant and carcinoma. Ocular and neurological manifestations may also occur. Malignant skin lesions such as squamous cell carcinoma may also occur. ${ }^{4}$

\section{Conclusions}

Since multiple cutaneous neoplasms develop in persons with Xeroderma Pigmentosum at a younger age, early diagnosis and management could save their lives. Genetic counseling should be offered for families. Follow-up care should be geared towards educating the patient and the parents about effective sun protection and early recognition of skin cancer. The treatment is aimed at education to minimize sun exposure and regular follow-ups for the early detection and treatment of skin cancers.

\section{References}

1. Lehmann AR, Mcgibbon D, Stefanini M. Xeroderma pigmentosum. Orphanet J Rare Dis 2011; 6(1): 70.

2. Naik SM, Shenoy AM, Rajshekar AN. Cutaneous Malignancies in Xeroderma Pigmentosum : Earlier Management Improves Survival. Indian J otolaryngol Head Neck Surg 2013;65:162-7.

3. Hasan S, Saeed S., 2015. Journal of Pigmentary Disorders Xeroderma Pigmentosum-A Rare Genodermatosis : Overview of Literature. J Pigment Disord $2015 ; 2: 230$.

4. Anand B, Kailasam S, Kumar PM, Srividhya K. Xeroderma Pigmentosum: A Rare Case Report with Review of Literature. J Indian Aca Oral Med Radiol 2017; 24(4):334-7.

5. Moriwaki S, Kanda F, Hayashi M, Yamashita D. Xeroderma pigmentosum clinical practice guidelines. J Dermatol 2017; 4(10): 1087-96.

6. Chan C, Section I, Chase C., 
2012.Ophthalmic Manifestations and Histopathology of Xeroderma Pigmentosum: Two Clinicopathological Cases and a Review of the Literature. Surv Ophtalmol 2011;56(4): 348-61.

7. Kaloga M, Diatta BA, Bammo M, Kourouma S, Diabate A, Gueye N, et al., 2016. Squamous Cell Carcinoma in African Children with Xeroderma Pigmentosum: Three Case Reports.
Case Rep Dermatol 2016;8(3): 311-8. . 8. Odhiambo AO, Kaminja K, Munyao PT., 2015. Case Report Suspected case of stage 3 Xeroderma pigmentosa : a case report. Afr J Haematol Oncol 2015;5:15-8.

9. Sethi M, Lehmann AR, Fassihi H. Xeroderma Pigmentosum: A Multidisciplinary Approach. EMJ Dermatol 2013;1: 54-63.
10. Thomas J, Tamilarasi S, Najeeb N, Manoharan D. Xeroderma Pigmentosum With Squamous Cell Carcinoma: A Case Report. Int J Adv case reports 2015;2(14): 883-5.

11. Lambert WC, Lambert MW. Development of Effective Skin Cancer Treatment and Prevention in xeroderma pigmentosum. Photochem photobiol 201;91(2):475-83. 